\title{
A Study of Meson Correlators at Finite Temperature*
}

QCD-TARO Collaboration: Ph. de Forcrand ${ }^{\mathrm{a}}$, M. García Pérez ${ }^{\mathrm{b}}$, T. Hashimoto $^{\mathrm{c}}$, S. Hioki $^{\mathrm{d}}$, R. Katayama ${ }^{e}$, H. Matsufuru ${ }^{f}$, O. Miyamura ${ }^{e}$, A. Nakamura ${ }^{g}$, I.-O. Stamatescu ${ }^{\text {h,i }}$, T. Takaishi ${ }^{j}$, and T. Umeda ${ }^{e}$

${ }^{a}$ ETH-Zürich, CH-8092 Zürich, Switzerland

${ }^{\text {b}}$ Dept. Física Teórica, Universidad Autónoma de Madrid, E-28049 Madrid, Spain

${ }^{\mathrm{c}}$ Dept. of Appl. Phys., Fac. of Engineering, Fukui Univ., Fukui 910-8507, Japan

${ }^{\mathrm{d}}$ Dept. of Physics, Tezukayama Univ.,Nara 631-8501, Japan

e Dept. of Physics, Hiroshima Univ., Higashi-Hiroshima 739-8526, Japan

${ }^{\mathrm{f}}$ Research Center for Nuclear Physics, Osaka Univ., Ibaraki 567-0047, Japan

${ }^{\mathrm{g}}$ Res. Inst. for Inform. Sc. and Education, Hiroshima Univ., Higashi-Hiroshima 739-8521, Japan

${ }^{\mathrm{h}}$ Institut für Theoretische Physik, Univ. Heidelberg D-69120 Heidelberg, Germany

${ }^{\mathrm{i}}$ FEST, Schmeilweg 5, D-69118 Heidelberg, Germany

${ }^{\mathrm{j} H i r o s h i m a ~ U n i v e r s i t y ~ o f ~ E c o n o m i c s, ~ H i r o s h i m a ~ 731-01, ~ J a p a n ~}$

We present results for mesonic propagators in temporal and spatial directions at $T$ below and above the deconfining transition in quenched QCD. Anisotropic lattices are used to get enough information in the temporal direction. We use the Wilson fermion action for light quarks and Fermilab action for heavy quarks.

\section{Introduction}

Hadronic properties are expected to change with increasing temperature, especially near to and above the QCD phase transition. Forthcoming heavy ion collision experiments will give the opportunity to investigate them, and require theoretical studies of signals of the phase transition and the QGP formation. Lattice QCD simulations allow us to perform model independent nonperturbative studies.

So far most calculations of hadronic masses at finite temperature have handled only the screening mass [1] but not the pole mass, which has a fundamental importance. In addition to the intrinsic ambiguity in defining pole masses at finite temperature the main difficulty comes from the shortness of the "temporal" (temperature) extension, $l_{\tau}=1 / T$.

Here, we attack the problem by using optimised hadronic operators on anisotropic lattices. Our strategy is as follows:

(1) to use anisotropic lattices to obtain a good resolution in the temporal direction.

\footnotetext{
*Poster by T. Umeda at LATTICE99.
}

(2) to seek for a good operator which has a large overlap with the state of interest at zero temperature.

(3) to observe temperature dependent effects by using this optimised operator on finite temperature lattices.

In the following, we report results on light and heavy mesons. Since part of the former have already been reported [2], we briefly summarise the current results. On the latter, we describe our computation and preliminary results.

\section{Results on light mesons at finite tem- perature}

We use lattices of $12^{3} \times N_{\tau}$ with $N_{\tau}=72,20,16$ and 12 at $\beta=5.68, \gamma_{G}=4$, in the quenched approximation 2 . Calibration using Wilson loops at $N_{t}=72$ gives the anisotropy $\xi=a_{\sigma} / a_{\tau}=5.3(1)$ and $a_{\sigma}^{-1}=0.85(3) \mathrm{GeV}$. Our lattices correspond to temperatures $T \simeq 0,0.93 T_{c}, 1.15 T_{c}$ and $1.5 T_{c}$ respectively.

For light quarks, anisotropic Wilson fermions

${ }^{2}$ The latticementioned in this report corresponds to "SetB" data in [2] 
are employed. After Coulomb gauge fixing, the PS meson is measured by the following operators;

$$
\begin{array}{r}
G_{P S}(r, t)=\sum_{\overrightarrow{m_{1}}, \overrightarrow{m_{2}}, \vec{n}} w\left(\overrightarrow{m_{1}}\right) w\left(\overrightarrow{m_{2}}\right) \\
<\operatorname{Tr} M\left(\overrightarrow{m_{1}}, 0 ; \vec{n}, t\right) M\left(\overrightarrow{m_{2}}, 0 ; \vec{n}+\vec{r}, t\right)^{\dagger}>
\end{array}
$$

where $M\left(\vec{m}, m_{0} ; \vec{n}, n_{0}\right)$ is a quark propagator and $w(\vec{m})$ is $\exp \left(-\alpha|\vec{m}|^{p}\right)$, which we call "exp-source" here. After tuning the parameters $\alpha$ and $p$, the correlator at $T=0$ is dominated by the lowest state [2]. Then the correlators at finite temperature are measured: It turns out that the effective masses change significantly above $T_{c}$, while $T \simeq 0.93 T_{c}\left(N_{t}=20\right)$ reveals almost the same features as $T=0$.

We also examine the spatial structure of the correlator above $T_{c}$. In Fig. 11 we compare the $P s$ "wave functions" normalised at $x=0$, $G_{P s}(x, t) / G_{P s}(0, t)$, at several $t$ for $T \simeq 0.93 T_{c}$ and $T \simeq 1.5 T_{c}$. Also shown is the free quark case $\left(m_{q} a_{\sigma}=0.1, \gamma_{F}=\xi=5.3\right)$. The $\exp$-exp source appears somewhat too broad at $T \simeq 0.93 T_{c}$ : the quarks come close to each other while propagating in $t$. An unexpected fact is that this is also the case at $T \simeq 1.5 T_{c}$ : the spatial distribution shrinks and stabilises, indicating that even at this high temperature there is tendency for quark and anti-quark to stay together. This is in clear contrast to the free quark case which never shows such a behaviour regardless of the source. The same feature holds for the other mesons at all quark masses.

Fig. 2 shows the temperature dependence of "masses" which are extracted from the "exp-exp" correlators at the three points $\left[N_{t} / 2-2, N_{t} / 2\right]$. Though the values above $T_{c}$ have large systematic errors, pole masses indicate chiral restoration there. We also show in the figure the screening masses: their difference with the pole masses is manifest. It is also noted that mesons degenerate approximately irrespective of their spin.

\section{Results on heavy quark systems at finite temperature}

Our target is mainly charmonium physics, e.g. investigations of the mass shift near $T_{c}[3]$ and $J / \psi$ suppression in the plasma phase 伯. In or-

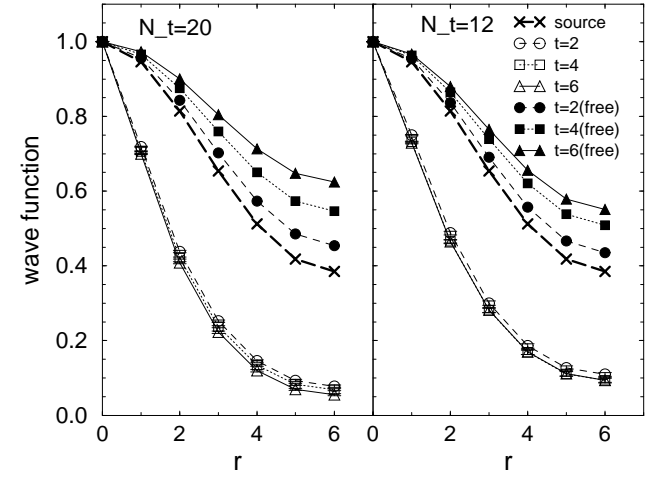

Figure 1. Ps wave function $\left(\kappa_{\sigma}=0.086\right.$, exp-exp source) normalised at $r=0$. Also plotted is the initial distribution of separations as given by the source. $T \simeq 0.93 T_{c}$ (left) and $T \simeq 1.5 T_{c}$ (right).

der to treat heavy quarks with the currently available computer power, we use the effective theory approach. We adopt Fermilab action with $O(a)$ improvement [5]. On the anisotropic lattice, the heavy quark operator is $\beta$ :

$K(x, y)=\delta_{x, y}-\kappa_{\tau}\left\{\left(1-\gamma_{4}\right) T_{+4}+\left(1+\gamma_{4}\right) T_{-4}\right\}$

$-\kappa_{\sigma} \sum_{i}\left\{\left(r-\gamma_{i}\right) T_{+i}+\left(r+\gamma_{i}\right) T_{-i}\right\}$

$-r\left(\kappa_{t} c_{E} g \sigma_{4 i} F_{4 i}(x)+\kappa_{s} c_{B} \frac{1}{2} g \sigma_{i j} F_{i j}(x)\right) \delta_{x, y}$.

where, $T_{ \pm \mu}=U_{ \pm \mu}(x) \delta_{x \pm \hat{\mu}, y}, \kappa_{s}=1 / 2(m+\xi+$ $3 r), \kappa_{t}=\xi \kappa_{s}$, and $r=1 / \xi$. Mean-field improvement is done for $c_{E}$ and $c_{B}$.

Introducing the anisotropy results in changing the free quark dispersion relation. In eq.(2), one notices that a larger anisotropy $\xi$ causes a smaller Wilson term. Then the problem is how the contribution from the momentum region at the edge of the Brillouin zone becomes larger. In the heavy quarkonium, typical energy and momentum scales exchanged in the meson are of order $m v^{2}$ and $m v$ respectively. For the charmonium, $v^{2} \sim 0.3$, and the typical energy scale is $\sim 500$ $\mathrm{MeV}$. Let us consider our lattice: $a_{\sigma}^{-1}=0.85 \mathrm{GeV}$ and $\xi=5.3, m \sim 0.3$ roughly corresponds to the charm quark mass. From the dispersion relation,

\footnotetext{
${ }^{3}$ This form differs from that of 6
} 


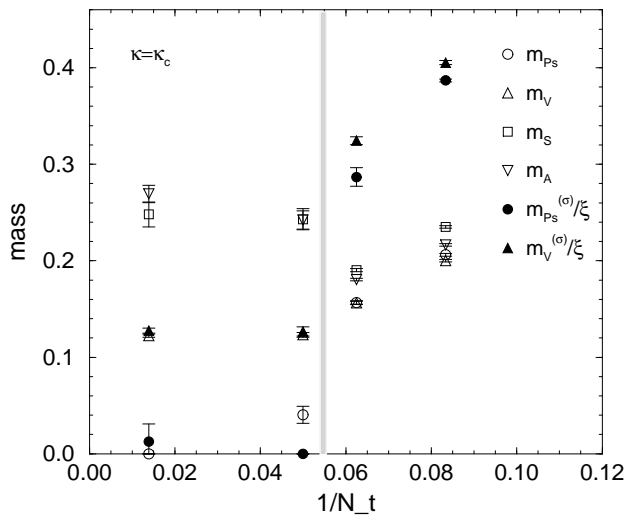

Figure 2. Temperature dependence of the mass $m$ (open symbols) and screening mass $m^{(\sigma)}$ (full symbols) in units $a_{\tau}^{-1}$ in the chiral limit. The vertical gray lines indicate $T_{c}$. The data correspond to $T \simeq 0,0.93 T_{c}, 1.15 T_{c}$ and $1.5 T_{c}$.

$E\left(p_{z}=a / \pi\right)-E(0) \sim 0.5 \mathrm{GeV}$. Though this result seems not sufficient, the effect is expected to be small for the ground state. With increasing lattice cutoff, this problem becomes milder.

We used the same configurations as for the light meson study. The anisotropy $\xi$ is fixed by using the dispersion relation. At present one set of parameters is available: $\kappa_{\sigma}=0.0984$ and $\gamma_{F}=3.67$ which corresponds to the vector meson mass $m_{V} \sim 3.10 \mathrm{GeV}$.

Fig.3 shows the effective masses of $P s$ and $V$ mesons at finite temperature. As for light mesons, on the $N_{\tau}=20$ lattice the lowest vector and pseudoscalar states keep almost the same masses as at $T=0$. Above $T_{c}$ we observe, as in the light meson case, that the $\mathrm{V}$ meson effective mass goes below the Ps meson. However, the Ps effective mass stays at almost the same position as at $T=0$ while the $\mathrm{V}$ effective mass decreases fast, in contrast to the light meson case where both of them increase with $T$.

\section{Summary and Outlook}

For a light quark system, the wave function indicates a strong correlation between quark and antiquark even above $T_{c}$. The pole masses show chiral restoration above $T_{c}$ and a behaviour quite different from that of the screening masses. For

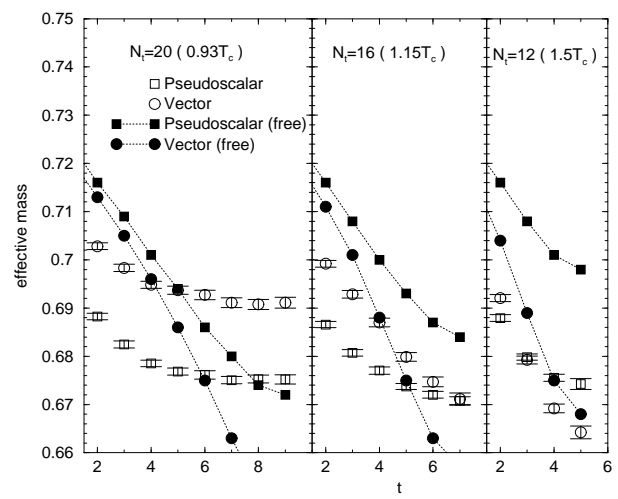

Figure 3. Effective mass with $\kappa_{\sigma}=0.0984$ and $\gamma_{F}=3.67$. At $N_{\tau}=72$ Pseudoscalar and Vector meson mass are $0.6756(7)$ and $0.6890(8)$ respectively. Full symbols show effective masses constructed by free quarks

heavy quark systems we use the Fermilab action on an anisotropic lattice. The effective mass of the vector meson drops sharply above $T_{c}$. Further investigations with finer lattices are undergoing.

The calculations have been done Intel Paragon $\mathrm{XP} / \mathrm{S}$ at INSAM, Hiroshima Univ. This work is supported by the Grant-in-Aide for Scientific Research by Monbusho,Japan (No 10640272, No 11440080).

\section{REFERENCES}

1. C.DeTar and J.Kogut, Phys. Rev. D36 (1987) 2828; C.DeTar, Phys. Rev. D36 (1988) 2328.

2. QCD-TARO: Ph. de Forcrand et al., Nucl. Phys. B(Proc. Suppl.) 73 (1999) 420: heplat/9809173; QCD-TARO: Ph. de Forcrand et al., hep-lat/9901017

3. T. Hashimoto et al., Phys. Rev. Lett. 57 (1986) 2123-2126.

4. T. Matsui, H. Satz, Phys. Lett. B178 (1986) 416-422.

5. A. El-Khadra, A.S. Kronfeld and P.B. Mackenzie, Phys. Rev. D55 (1997) 3933-3957.

6. T.R. Klassen, Nucl. Phys. B(Proc. Suppl.) $\mathbf{7 3}$ (1999) 918-920: hep-lat/9809174 